\title{
A Survey on Remotely Operated Quadrotor Aerial Vehicle using the Camera Perspective
}

\author{
Debadatta Sahoo \\ Dept. of EEE \\ Dr. M.G.R. University
}

\author{
Amit Kumar \\ Dept.of EEE \\ Dr. M.G.R. University
}

\author{
K. Sujatha \\ Assistant Professor \\ Dept. of EEE \\ Dr M.G.R University
}

\begin{abstract}
This survey paper presents a mission-centric approach to controlling the optical axis of a video camera mounted on a camera manipulator and fixed to a quad rotor remotely operated vehicle. A four-DOF quad rotor, UAV model will be combined with a two-DOF camera kinematic model to create a single system to provide a full six DOF actuation of the camera view. This survey work proposed exploits that all signals are described in camera frame. The closed-loop controller is designed based on a Lyapunov-type analysis so that the tracking result is shown to produce Globally Uniformly Ultimately Bounded (GUUB). Computer simulation results are provided to demonstrate the suggested controller. [1]
\end{abstract}

\section{Keywords}

Using MATLAB, dc brushless motor, remote control, manual control, visual camera.

\section{INTRODUCTION}

The typical scenario for using the quad rotor helicopter (or any aerial vehicle) as a video camera platform is based on mounting the camera on a positioned that is controlled independently from the vehicle. When the navigation or surveillance tasks become complicated, two people may be required to achieve the camera targeting objective: a pilot to navigate the UAV and a camera operator. An important underlying action on the part of the camera operator that makes this scenario feasible is that the camera operator must compensate for the motions of the UAV that disturb the camera targeting; uncompensated camera platform motion on the camera axis might be loss of targeting, but a scenario where the camera positioned is used to compensate for the platform motion can maintain the camera view. The potential shortcomings of this typical operational scenario can be summarized as: i) multiple skilled technicians are typically required, ii) the camera operator must compensate for the actions of the pilot, and iii) it is not intuitive for a camera operator to split the camera targeting tasks between actions of the camera positioned controlled by the operator and commands to the pilot. The problem of providing an intuitive interface with which an operator can move a camera positioned to make a video camera follow a target image appears in many places. The difficulty of moving a system that follows a subject with a video camera was recently addressed in. A die rent perspective to this same basic camera targeting problem was presented where the camera platform, a quad rotor UAV, and the camera positioning unit are considered to be a single robotic unit. The work in builds on to show the design of a velocity controller for the combined quad rotor-camera system that works from operator commands generated in the camera field-of-view to move both elements. The paper is organized as follows. In Section
3, a kinematic and dynamic model of the quad-rotor is presented. The kinematics for a three-link camera positioned are developed; however, only two links are used in any position scenario. The case of this positioned used in a 2-link, Tilt-Roll configuration to look forward is carried through the control design and simulation [2].

\section{LITERATURE REVIEW}

\subsection{Quadrotor model}

A. Under actuated Quad rotor Aerial Vehicle Model. The elements of the quad-rotor unmanned aerial vehicle model are shown in Figure 2. The quad rotor body fixed frame, F, is chosen to coincide with the center of gravity which implies that it has a diagonal inertia matrix. The kinematic and a dynamic model of a quad rotor are expressed as follows [1,2]

$$
\begin{gathered}
p_{i f}^{i}=R_{f}^{i}(\theta) v_{i f}^{f} \\
\theta_{i f}^{i}=T_{f}^{i}(\theta) \omega_{i f}^{f} \\
R_{f}^{i}=R_{f}^{i} s\left(\omega_{i f}^{f}\right) \\
m v_{i f}^{i}=F_{f}^{F}-m s\left(\omega_{i f}^{f}\right) v_{i f}^{f}+N_{1}\left(v_{i f}^{i}\right)+G\left(R_{f}^{i}\right)(4)
\end{gathered}
$$

In this model $v_{i f}^{i}(\mathrm{t})=[v x, v y, v z] \in R^{3} \mathrm{R}_{3}$ denotes the linear velocity of the quad rotor body-fixed frame $F$ with respect to the earth-fixed inertial frame, I, expressed in the body-fixed frame, $\mathrm{F}$, and $\omega_{i f}^{f}(t)=[\omega x, \omega y, \omega z] \in R^{3} \mathrm{R} 3$. denotes the angular velocity of the quadrotor body-fixed frame $\mathrm{F}$ with respect to the inertial frame, I, expressed in the body-fixed frame, F. Equations (1) - (3) represent the kinematics of the quad rotor. The $p_{i f}^{i}(t)$ in (1), is the velocity of the quad rotor and $\theta_{i f}^{i}$ in (2) represents, the angular velocity $\omega_{i f}^{f}(t)$ transformed by the matrix $T_{f}^{i}(\theta)$ The position and angle $p_{i f}^{i}(t), \theta_{i f}^{i}(t), \omega_{i f}^{f}(t), v_{i f}^{f}(t)$ are assumed to be measurable. Equation (2) represents the modeling assumption that angular velocity of the quad rotor is calculated directly in lieu of modeling the angular dynamics; that is $\omega_{i f}^{f}(t)$, is considered as the system input. The dynamics of the translational velocity is shown in (4) and contains the gravitational term, $\mathrm{G}\left(R_{f}^{i}\right)=m g\left(R_{f}^{i}\right) \mathrm{E} 3 \in \mathrm{R} 3$, where $\mathrm{g} \in \mathrm{R}$, denotes gravitational acceleration, $\mathrm{E} 3=[0,0,1]^{\mathrm{T}}$. denotes the unit vector in the coordinates of the inertial frame, $m \in \mathrm{R}^{1}$ is the known mass of the quad-rotor, $\mathrm{N}^{1}\left(v_{i f}^{i}\right) \in \mathrm{R}^{3}$. represents a bounded function, e.g., aerodynamic damping force, and $\mathrm{S}(.) \in$ $\mathrm{R}^{2 * 3}$. is a general form of the skew-symmetric matrix [6]. The 
quad rotor has inherently six degrees-of-freedom; however, the quad rotor has only four control inputs: one translational

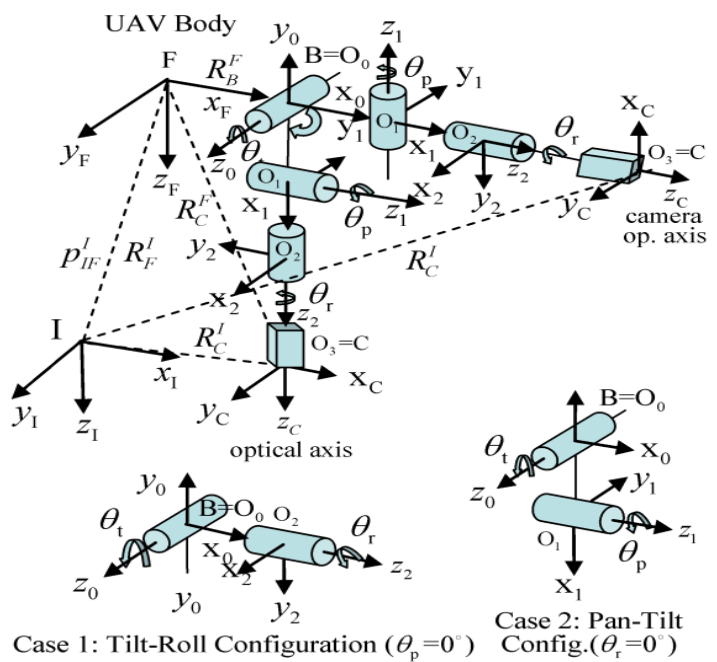

Figure 1. Quad rotor with a Pan-Tilt-Roll Camera Positioned

force along the z-axis and three angular velocities. The vector $F_{f}^{F}(\mathrm{t}) \in \mathrm{R}^{3}$ refers to the quad rotor translational forces but in reality represents the single translational force which is created by summing the forces generated by the four rotors and is expressed as

$$
F_{f}^{F}=\mathrm{B}_{1} \mathrm{U}_{1}\left[\begin{array}{ll}
0 & 0
\end{array}\right.
$$

where $\mathrm{B} 1=\mathrm{I} 3$ is a configuration matrix (actuator dynamics are beyond the scope of this design) and $\mathrm{u} 1(\mathrm{t}) \in \mathrm{R} 1$
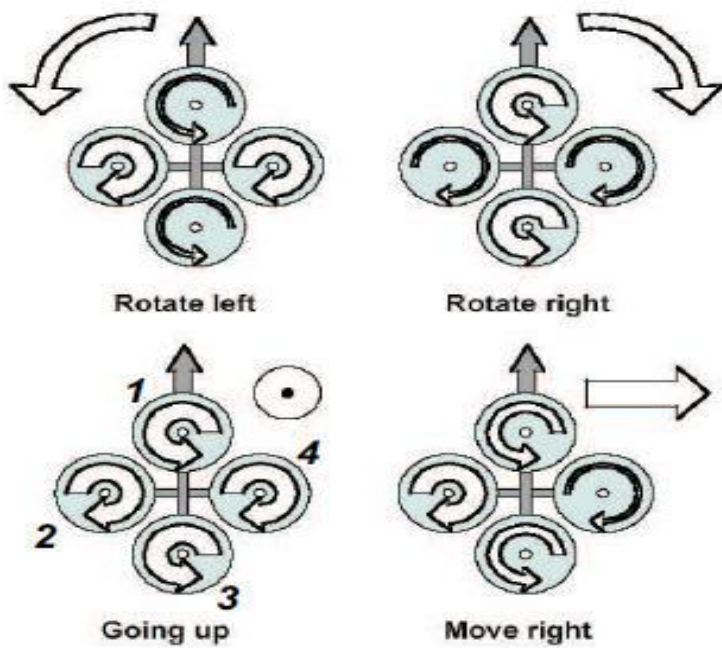

Figure2. Movement of quad rotor

A quad rotor has four motors located at the front, rear, left, and right ends of a cross frame. The quad rotor is controlled by changing the speed of rotation of each motor. The front and rear rotors rotate in a counter-clockwise direction while the left and right rotors rotate in a clockwise direction to balance the torque created by the spinning rotors. The relative speed of the left and right rotors is varied to control the roll rate of the UAV. Increasing the speed of the left motor by the same amount that the speed of the right motor is decreased will keep the total thrust provided by the four rotors approximately the same. In addition, the total torque created by these two rotors will remain constant. Similarly, the pitch rate is controlled by varying the relative speed of the front and rear rotors. The yaw rate is controlled by varying the relative speed of the clockwise (right and left) and counter-clockwise (front and rear) rotors. The collective thrust is controlled by varying the speed of all the rotors simultaneously.[1-3],[4]

\subsection{Camera Positioned Kinematics}

As stated, the quad-rotor can thrust in the z-direction, but it cannot thrust in the $\mathrm{x}$ - or $\mathrm{y}$-directions. Since the quad rotor helicopter is under actuated in two of its translational velocities, a two actuator camera is added to achieve six degrees of freedom (DOF) control in the camera frame. A tiltroll camera is added to the front of the helicopter as seen in. With the new camera frame, there are now three rotations and three translations, a total of six DOF, to actuate. To control any of the DOF, either the camera must move, the UAV must move, or both.

\subsubsection{Tilt-Roll Camera on Front of UAV}

The rotation matrix between UAV frame and Camera frame seen in upper Fig 1 is:

$$
R_{c}^{f}=\left(\begin{array}{ccc}
\sin \theta_{\mathrm{t}} \cos \theta_{\mathrm{r}} & \sin \theta_{\mathrm{t}} \sin \theta_{\mathrm{r}} & \cos \theta_{\mathrm{t}} \\
\sin \theta_{\mathrm{r}} & \cos \theta_{\mathrm{r}} & 0 \\
-\cos \theta_{\mathrm{t}} \cos \theta_{\mathrm{r}} & \cos \theta_{\mathrm{t}} \sin \theta_{\mathrm{r}} & -\sin \theta_{\mathrm{t}}
\end{array}\right)
$$

Since only two of the angles vary, the Jacobian can be redefined as

$$
\mathrm{J}_{\mathrm{C}(\text { front })}=\left(\begin{array}{cc}
0 & \cos \theta \\
1 & 0 \\
0 & \sin \theta
\end{array}\right)
$$

and finally

$$
\omega_{f c}^{f}=\mathrm{j}_{\mathrm{c}(\text { front })} \Theta_{\mathrm{c}}, \Theta_{\mathrm{c}}=\left(\Theta_{\mathrm{t}} \Theta_{\mathrm{r}}\right]^{\mathrm{t}} \in R^{2}
$$

which facilitates the calculation of the angles of the camera.[02]

\subsubsection{Visual sensor}

Typically, the visual sensor consists of a camera and image processing block. In the simulation the object was defined as $3 \mathrm{x} 1$ vectors of coordinates related to earth for each points by 'polyhedral' command in MATLAB. To characterize the object four feature points were selected, being defined as the camera was modelled by using the positions and orientations of the camera and the object $(\boldsymbol{x} \boldsymbol{c}, \boldsymbol{x o})$. The image processing block is modelled in the details of imaging geometry and perspective projection can be found in many computer vision texts [6]. To develop the visual sensor model, first the frames are defined. The helicopter frame is $\mathbf{R h}$, the camera frame is $\mathbf{R c}$ and the object frame is Ro as shown in Figure 3. 


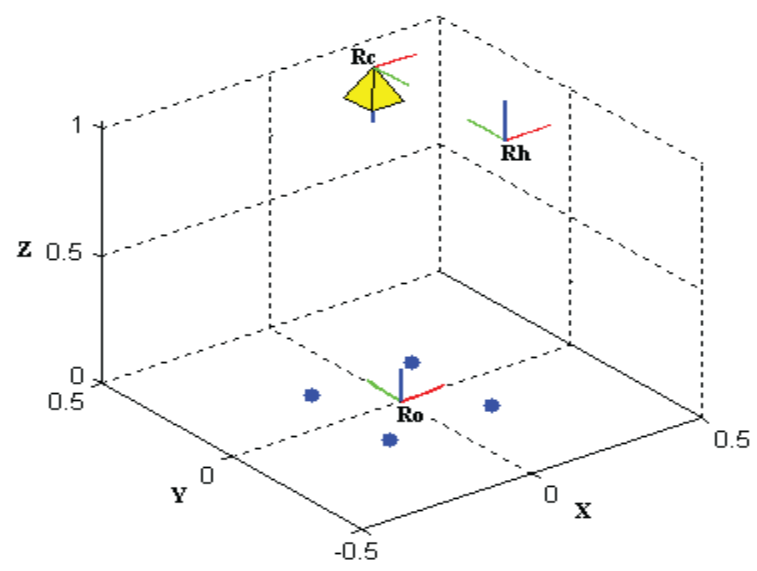

Figure3. The axes of the camera object and helicopter

\subsection{Control of quadrotor}

The helicopter controllers have four input commands as U1, $\mathrm{U} 2, \mathrm{U} 3$, and $\mathrm{U} 4$. U1 represents the translation around the $\mathrm{z}$ axis. U2 represents the rotation around the $y$ axis (roll angle). $\mathrm{U} 3$ represents the rotation around the $\mathrm{x}$ axis (pitch angle). Finally, U4 represents the rotation around the $\mathrm{z}$ axis (yaw angle). In this study, Proportional-Derivative (PD) controllers are designed to control the helicopter [6]. This is because that the control algorithm can be obtained from the helicopter model and this algorithm makes the system exponentially stable as

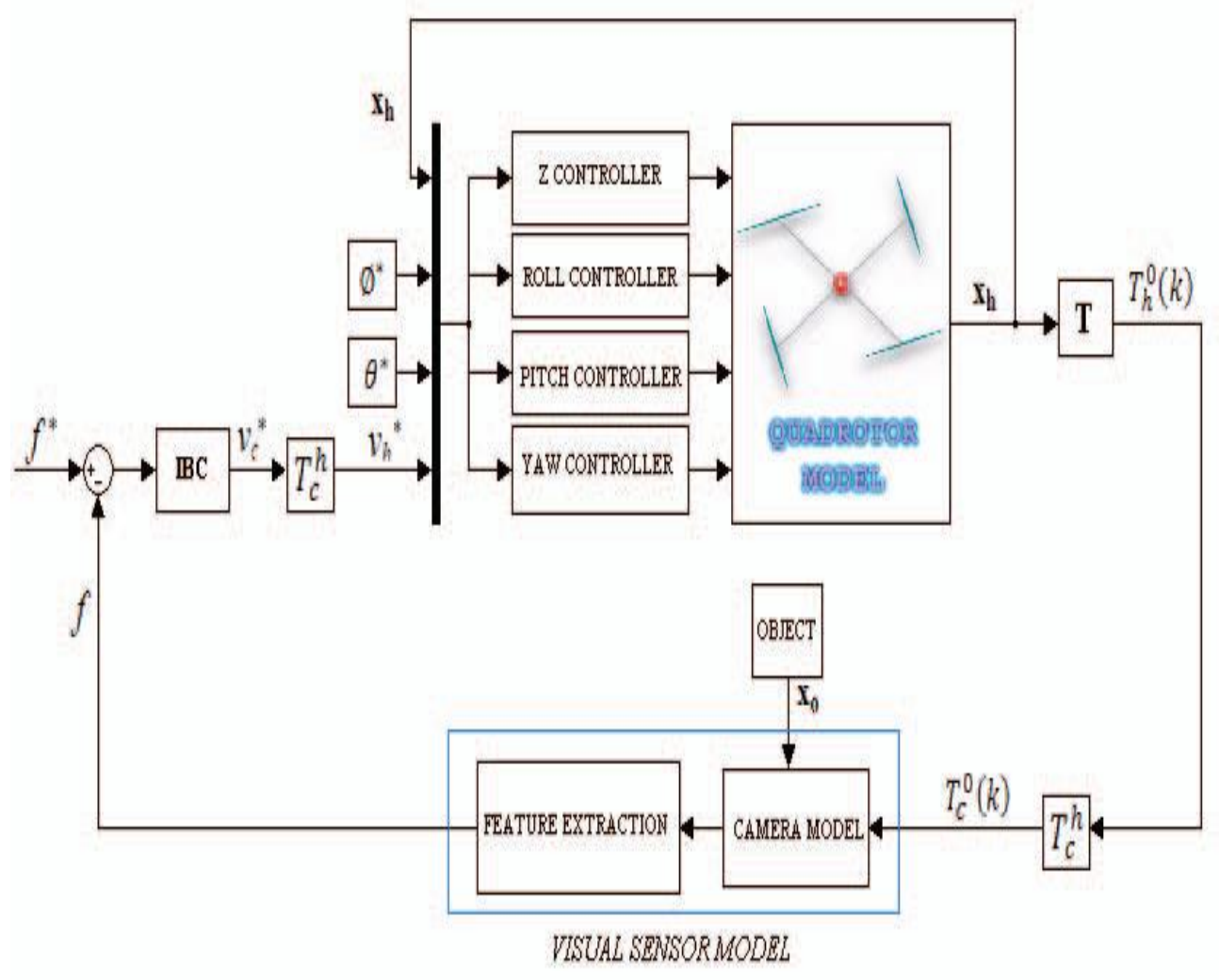

Figure 4. Control system of quadrotor

\subsubsection{Altitude Control}

For the altitude control of the helicopter Equation 1 is used

$$
\mathrm{U}_{1}=\frac{m g}{\cos \theta \cos \emptyset}+m \frac{[k d(z-\dot{z})]}{\cos \theta \cos \emptyset}
$$

where, $\mathrm{z}^{*}$ is the reference linear velocity value around the $\mathrm{z}$ axis which is the third component of helicopter reference velocity vector $\mathrm{vh}^{*}$.

\subsubsection{Translation Control}

It is necessary to control the pitch and roll angles for controlling the translations around the $\mathrm{x}$ and $\mathrm{y}$ axis. Therefore, for translation around $\mathrm{x}$ axis, reference pitch angle and angular rate of pitch angle $\left(\theta^{*}, \Theta^{*}\right)$ are demanded. In the same way, for translation around y axis, reference roll angle $\left(\varphi^{*}, \varphi\right.$ $*$ ) and angular rate of roll angle are demanded. While the angular rates are determined from $v h^{*}$ vector $\left(4^{\text {th }}\right.$ and $5^{\text {th }}$ components), the angles are determined by using Eq (2).

$$
\begin{aligned}
& \varphi^{*}=\arcsin \left[\mathrm{kd}_{\mathrm{y}}\left(\mathrm{y}^{*}-\mathrm{y}^{\prime}\right)\right] \\
& \varphi^{*}=\arcsin \left[\mathrm{kd}_{\mathrm{x}}\left(\mathrm{x}^{*}-\mathrm{x}^{*}\right)\right] \\
& \mathrm{U}_{2}=\mathrm{kp} \varphi\left(\varphi^{*}-\varphi\right)-k d \varphi \varphi \\
& U_{3}=k p \Theta\left(\Theta^{*}-\Theta\right)-\mathrm{kd} \Theta \Theta
\end{aligned}
$$

\subsubsection{Yaw Control}

Desired input signal for the yaw control of the helicopter is presented in equation 3

$$
\mathrm{U}_{4}=\operatorname{kd} \varphi\left(\varphi^{*}-\varphi^{\prime}\right)
$$



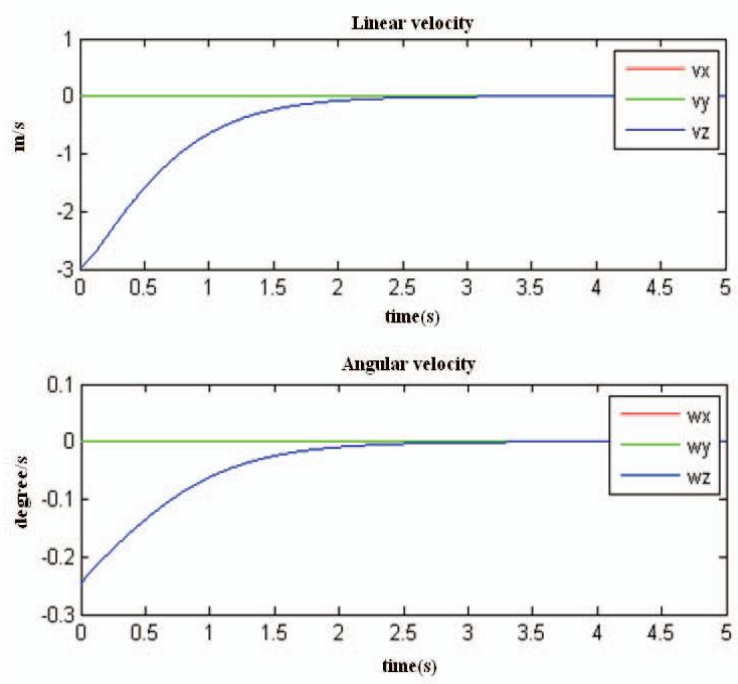

Figure 5. Linear and angular velocities of the helicopter

\subsection{Design Procedure for quadrotor}

A custom designed experimental test stand shown in Figure 6 was used to perform secure experiments. The test stand allows the helicopter to perform yaw motions freely, allows up to 2 meters of altitude, as well as up to $\pm 20^{\circ}$ roll and pitch motion. The experiment system consists of a model quad rotor helicopter, a test stand, a pan/tit/zoom camera, a catadioptric camera to be used in the future researches, and an IMU sensor. A Core2Quad $2.40 \mathrm{GHz}$ processor desktop computer with 3 GBs RAM on Windows XP that has a dual framegrabber has been used. Algorithms were developed using Matrix Imaging Library 8.0 on C++ [20]. A Sony pan/tilt/zoom camera is directed to a stationary ground target. Captured images are processed with a feature extraction routine to determine the black blob features on the scene. Black blobs of various sizes were selected for simplicity and real-time performance. In order to show the effectiveness of the proposed algorithms an experiment of yaw motion under visual-servo control has been performed. The helicopter starts at 70 degree yaw angle and the goal is to reach 110 degree yaw angle under visual servo control. The Euler angles of the helicopter during the experiment are presented in Figure. The helicopter reaches the desired yaw values as the roll and pitch angles are kept at zero degrees during the motion.

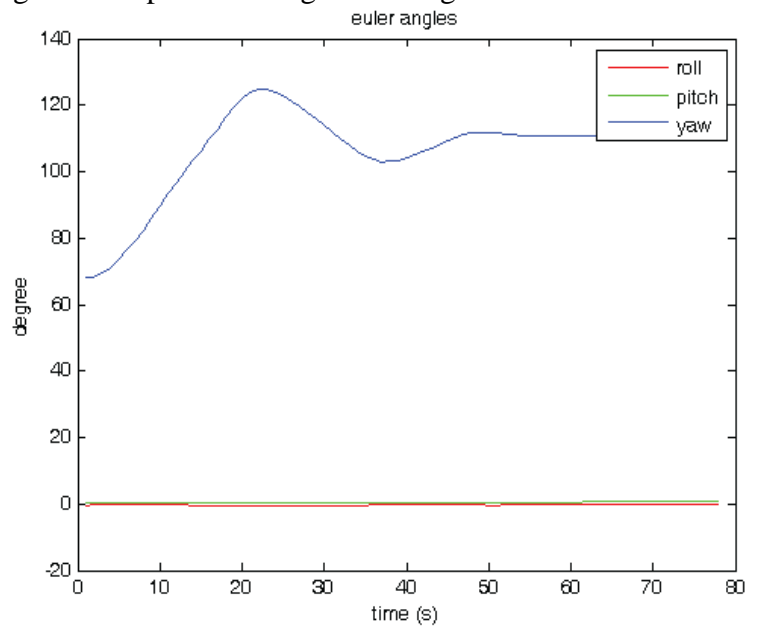

Figure 6. The Euler angles of the helicopter during the experiment.
The linear and angular velocities during the experiment are presented in Figure 6. The desired angular velocity which is related with the yaw motion approaches zero line as helicopter approaches the desired yaw angle.
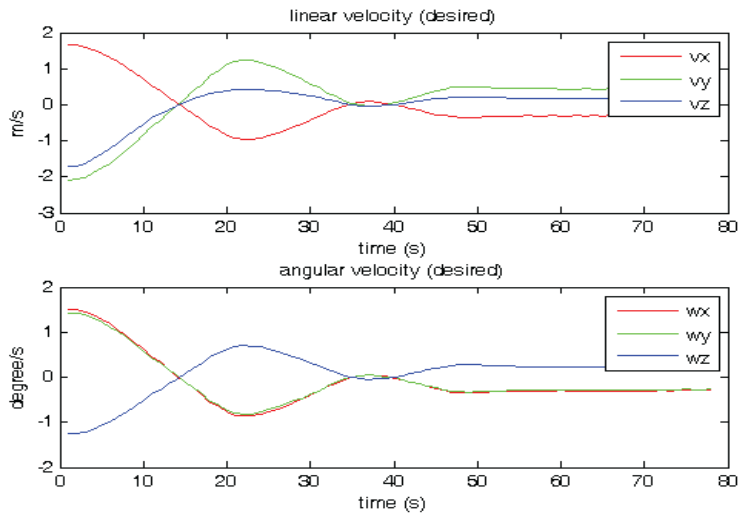

Figure7. Results of the yaw control experiment.

\section{PROPOSED TECHNIQUE}

The proposed techniques in our UAV are listed below .It has four rotor and using four propeller. It will be more stable. It has one vision camera. It can give the proper image up to $100 \mathrm{~m}$ fit (approximate). It can attain a height up to $100 \mathrm{fit}$ (approximate). It has brushless dc motor of capacity 3000 rpm, which will be very light in weight. Our project showcases important control capabilities which allow for autonomous balancing of a system which is otherwise dynamically unstable. A quad-rotor poses a more challenging control problem than a single-rotor or dual-rotor inline helicopter because the controls demands include accounting for subtle variations which exist between the motors and cause each motor to provide a slightly different level of lift. In order for the quad-rotor craft to be stable, the four motors must all provide the same amount of lift, and it is the task of the control system to account for variations between motors by adjusting the power supplied to each one. We deemed the control of a quad-rotor craft as a valuable challenge to pursue. The benefits of such a craft warrant the design challenges, as a quad-rotor craft is more efficient and nimble than a singlerotor craft. Unlike a single-rotor craft, which uses a second, smaller vertical propeller to change direction, the quad-rotor craft's directional motion is generated by the same four motors that are providing lift. Also, the quad-rotor can change direction without having to reorient itself - there is no distinction between front and back of the craft. In the quadrotor, every rotor plays a roll in direction and balance of the vehicle as well as lift, unlike the more traditional single rotor helicopter designs in which each rotor has a specific task - lift or directional control - but never both. We have use the structural component, mentioned in table 1 below.

Table 1. Structural Components

\begin{tabular}{|l|l|}
\hline S.no & Name of structural component \\
\hline 1 & Brush less dc motor \\
\hline 2 & Pusher propeller, Tractor propeller \\
\hline 3 & Gear box \\
\hline 4 & Arm \\
\hline 5 & Battery \\
\hline 6 & Central hub \\
\hline 7 & Motor mount \\
\hline
\end{tabular}




\begin{tabular}{|l|l|}
\hline 8 & Speed controller \\
\hline 9 & Visual camera \\
\hline
\end{tabular}

\subsection{Brushless DC Motor}

The motors are cobalt, brushed, DC motors rated for $12 \mathrm{~V}, 15$ amps. The DC, brushed motor configuration was desired for ease of control (ability to control via PWM). The cobalt motors use strong rare earth magnets and provide the best power to weight ratio of the hobby motors available for model aircraft. We were limited to these hobby motors by our design budget. As a result, the rest of our structural design revolves around the selection of these motors and the allowable weight of the craft based on the lift provided by these motors (approximately $350 \mathrm{~g}$ of lift from each motor) as shown in Figure 8.

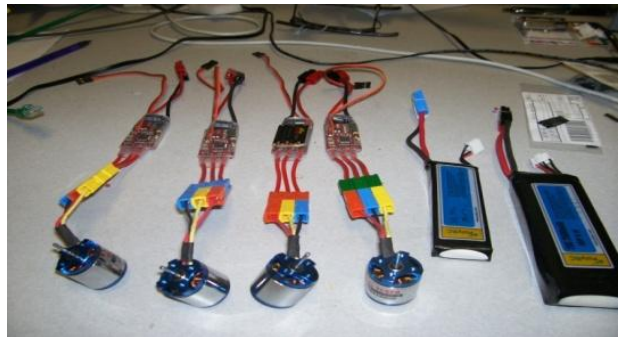

Figure 8. brushless dc motor and battery

\subsection{Propellers}

The propellers are 10" from tip to tip. Two are of the tractor style, for clockwise rotation, and the other two are of the pusher style, for counter clockwise rotation. For our design, a propeller with a shallow angle of attack was necessary as it provided the vertical lift for stable hovering. The propellers we used were steeper than the ideal design because of limited availability of propellers that are produced in both the tractor and pusher styles.

\subsection{Gearboxes}

The gearboxes have a 2.5:1 gear ratio. They reduce the speed of the prop compared to the speed of the motor, allowing the motors to exert more torque on the propellers while drawing much less current than in a direct drive configuration.

\subsection{Arms}

The arms of our quad-rotor design needed to be light and strong enough to withstand the 10 stress and strain caused by the weight of the motors and the central hub at their opposite ends. Carbon fibre was deemed the best choice because of its weight to strength ratio. The thickness of the tube was chosen to be the smallest possible to lower its weight. The length of each arm (10") was chosen based on the propellers. The propellers used are 10" long each so we had to allow enough room for them to spin without encountering turbulence from one another. Since such a phenomenon would be quite complex to analyze, we simply distanced the motors far enough apart to avoid the possibility of turbulence interference among rotors.

\subsection{Battery}

The battery was selected on the basis of power requirements for the selected motor/gearbox combination. We opted for a battery of the lithium polymer variety, despite the fact that it was considerably more expensive than other batteries providing the same power, because this battery provided the best power-to-weight ratio. Our battery choice was a 1450mah $12.0 \mathrm{~V} 12 \mathrm{C}$ Li-polymer battery. (Note: Because we did not have enough time to integrate the circuitry of the controls system on-board, and thus performed only tethered flight, we did not ultimately purchase the battery.) as shown in Figure 9.

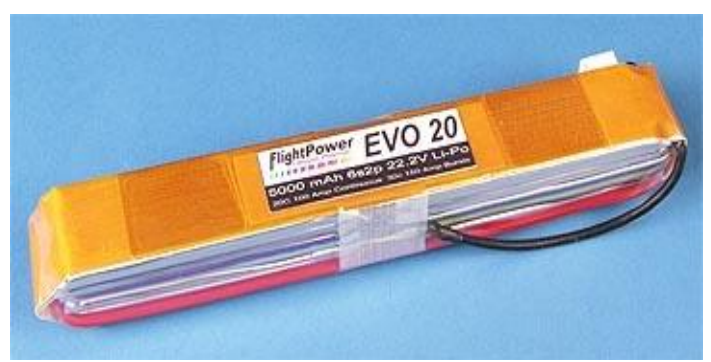

Figure 9. Lithium polymer variety

\subsection{Central Hub}

The central hub carries all of the electronics, sensors, and battery. It sits lower than the four motors in order to bring the centre of gravity downwards for increased stability. We manufactured it using a rapid prototyping machine considering our design for the hub, the rapid prototyping machine was ideal because of its ability to produce relatively complex details, for example the angled holes which allow for the central hub to sit lower than the surrounding motors. The thermoplastic polymer used in rapid prototyping has good strength to weight ratio.

\subsection{Motor Mounts}

The motor mounts connect the motors to the carbon fiber arms. Because of their complex details, they were manufactured using the rapid prototyping machine and therefore made of thermoplastic polymer.

\subsection{Block diagram of controlling quadrotor}

The following schematic depicts our controls system. The diagram represents how the control system interacts with the physical system for controlled quad-rotor flight. The control of the system involves four independent PID loops. A PID loop is need for pitch control, roll control, yaw control, and height control. As each of the PID controls calculates how the platform has to change, the results are summed up for each of the motors resulting in the correction needed for integrated control of the Quad Rotor as shown Figure 10.

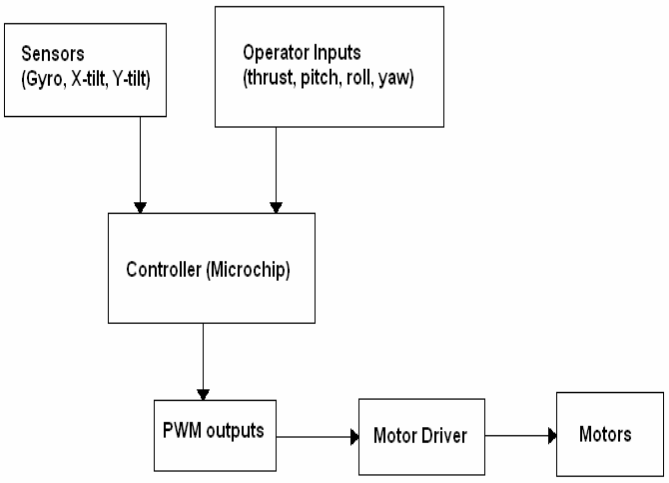

Figure 10. Block diagram Control system schematic 


\section{CONCLUSION}

This survey paper suggests a novel fly-the-camera approach to designing a nonlinear controller for an under actuated quad rotor aerial vehicle that compliments the quad rotor motion with two additional camera axes to produce a fully actuated camera targeting platform. The approach fuses the often separate tasks of vehicle navigation and camera targeting into a single task where the pilot sees and flies the system as through riding on the camera optical axis. The controller was shown to provide position and angle tracking in the form of Globally Uniform Ultimately Bounded (GUUB) result. Visual information has been used solely for the control of the vehicle with the feature estimation, image based control, and helicopter controller blocks. Various simulations in MATLAB and experiments performed on a model helicopter show that the approach is successful. As a future work, we plan to experimentally validate the simulation results with stationary and non-stationary objects in the control loop with more advanced motions.

\section{ACKNOWLEDGEMENTS}

The authors gratefully acknowledge the authorities of Dr. M.G.R. Educational and Research Institute, Chennai, India, for the facilities provided to carry out this research work. Many lives \& destinies are destroyed due to the lack of proper guidance, directions \& opportunities. It is in this respect we feel that we are in much better condition today due to continuous process of motivation \& focus provided by my parents \& teachers in general. The process of completion of this project was a tedious job \& requires care \& support at all stages. We would like to highlight the role played by the individuals towards this.

We are internally grateful to honorable Mrs. K.Sujatha for providing us the opportunity $\&$ infrastructure to complete the project as a partial fulfillment of B.Tech degree. We are very thankful to L.Ramesh, Head of Department of EEE, for his kind support \& faith in us.

\section{REFRENCES}

[1] Nicolas Guenard, Tarek Hamel,and Robert Mahony, "A Practical Visual Servo Control for an Unmanned Aerial Vehicle" transaction on robotics,vol-24,no-2,april2008 33. Member, IEEE.

[2] Andrew E. Neff, DongBin Lee, Vilas K. Chitrakaran, Darren M. Dawson and Timothy C. Burg "Velocity Control for a Quad-Rotor UAV Fly-By-Camera Interface" Clemson University.

[3] Dong Bin Lee1, Vilas Chitrakaran2, Timothy Burg1, Darren Dawson1, and Bin Xian "Control of a Remotely Operated Quad rotor Aerial Vehicle and Camera Unit Using a Fly-The-Camera Perspective" Proceedings of the 46th IEEE Conference on Decision and Control New Orleans, LA, USA, Dec. 12-14, 2007.

[4] Clinton Allison Mark Schulz "Build a Micro Air Vehicle (MAV) Analysis and design of an on-board electronic system for a Quad Rotor Micro Aerial Vehicle." ENGG4801 Thesis Project.

[5] Erding Altuk, James P. Ostrowski, Camillo J. Taylor "Quad rotor Control Using Dual Camera Visual Feedback" GRASP Lab. University of Pennsylvania, Philadelphia,PA 19104, USA. September 14-19, 2003.

[6]. Zehra Ceren, Erdinç Altu "Vision-based Servo Control of a Quad rotor Air Vehicle”, IEEE, 2006.

[7]. Engr. M. Yasir Amir 1, Dr. Valiuddin Abbass 2 "Modeling of Quadrotor Helicopter Dynamics" April 911, 2008 in KINTEX,

[8]. James F. Roberts, Timothy S. Stirling, Jean-Christophe Zufferey and Dario Floreano Ecole Polytechnique Fédérale de Lausanne (EPFL), Lausanne, 1015, Switzerland. "Quadrotor Using

[9]. Thae Su Aye, Pan Thu Tun, Zaw Min Naing, and Yin Mon Myint. "Development of Unmanned Aerial Vehicle Manual Control System". World Academy of Science, Engineering and Technology 42, 2008 